\title{
A Complete Readout Chain of the ATLAS Tile Calorimeter for the HL-LHC: from FATALIC Front-End Electronics to Signal Reconstruction
}

\author{
Sergey Senkin, on behalf of the ATLAS Tile Calorimeter System \\ Laboratoire de Physique de Clermont-Ferrand \\ Campus Universitaire des Cézeaux \\ 4 Avenue Blaise Pascal \\ 63178 Aubière cedex, France \\ Email: sergey.senkin@cern.ch
}

\begin{abstract}
The ATLAS Collaboration has started a vast programme of upgrades in the context of high-luminosity LHC (HL-LHC) foreseen in 2024. We present here one of the frontend readout options, an ASIC called FATALIC, proposed for the high-luminosity phase LHC upgrade of the ATLAS Tile Calorimeter. Based on a $130 \mathrm{~nm}$ CMOS technology, FATALIC performs the complete signal processing, including amplification, shaping and digitisation. We describe the full characterisation of FATALIC and also the Optimal Filtering signal reconstruction method adapted to fully exploit the FATALIC three-range layout. Additionally we present the resolution performance of the whole chain measured using the charge injection system designed for calibration. Finally we discuss the results of the signal reconstruction used on real data collected during a preliminary beam test at CERN.
\end{abstract}

\section{INTRODUCTION}

ATLAS [1] is one of two general-purpose experiments at the Large Hadron Collider (LHC) at CERN. It is designed to realise the full discovery potential and the vast range of physics opportunities provided by the LHC. The Tile Calorimeter (TileCal) [2] is the hadronic calorimeter covering the central region of the ATLAS detector, as can be seen on Fig. 1. It is essential for the measurement of hadrons, jets, tau leptons and missing transverse energy.

The upgrade of the LHC to the high-luminosity phase (HLLHC) [3] foreseen in 2024 will provide invaluable opportunities for the search for new physics beyond the Standard Model, as well as the detailed studies of the electroweak symmetry breaking mechanism and precise measurements of the properties of recently discovered Higgs boson. The ATLAS Collaboration has started an extensive programme of HL-LHC upgrades for every sub-detector, including TileCal. The current readout electronics of TileCal must be upgraded to comply with the new specifications aiming for the future operating conditions.

The ASIC described in this document, named Front-end ATlAs tiLe Integrated Circuit (FATALIC) [4], has been developed to fulfil the requirements of the HL-LHC upgrade.

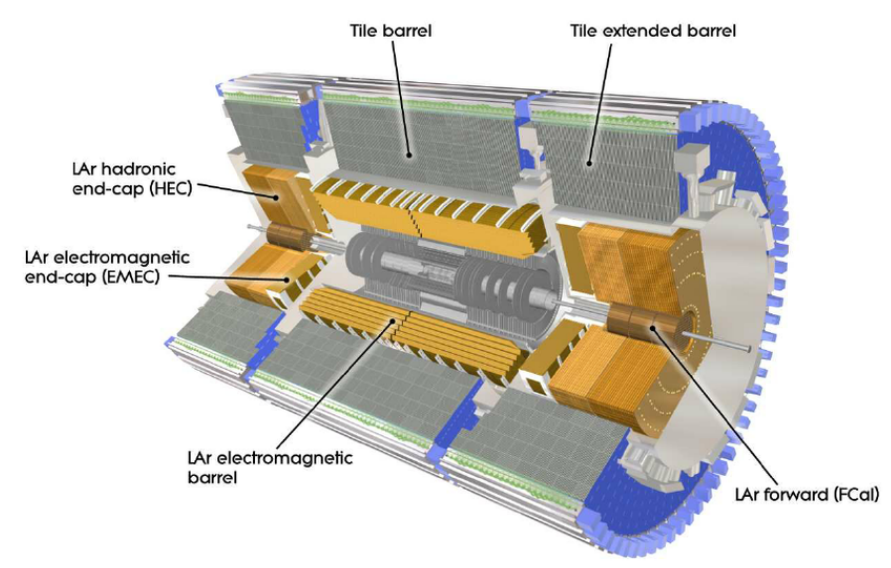

Fig. 1. A cut-away view of the ATLAS calorimeters. The TileCal comprises a central barrel region with pseudorapidity range of $|\eta|<1.7$, and two extended barrel regions providing coverage up to $|\eta|<2.4$.

It is one of the front-end readout options proposed for the upgrade of TileCal, based on a $130 \mathrm{~nm}$ CMOS technology and designed for the complete processing of the signal delivered by each Photomultiplier-Tube (PMT), including amplification, shaping and digitisation.

The document is structured as follows. The overview of the FATALIC layout as well as the main progress stages of its development and the performance measurements are given in Section II. The signal reconstruction aspects are described in Section III. Subsection III-A gives an overview of the Optimal Filtering algorithm which is used to reconstruct the amplitude and time of the signal is described. The specific details of application of this algorithm to FATALIC pulses are described in subsection III-B. Finally, the results of the signal reconstruction used on real data collected during a preliminary beam test at CERN are shown in Section IV, and a short conclusion is given in Section $\mathrm{V}$. 


\section{FATALIC LAYOUT}

The front-end readout system designed for the HL-LHC upgrade has to abide by stringent requirements, an overview of which is given in Table I. In order to comply with them and particularly with the large dynamic range requirement of $25 \mathrm{fC}$ to $1.2 \mathrm{nC}$, a three-gain layout has been adopted for FATALIC.

TABLE I

HL-LHC REQUIREMENTS FOR THE FRONT-END READOUT

\begin{tabular}{|c|c|}
\hline Technology & $0.13 \mu \mathrm{m}$ CMOS GlobalFoundries \\
\hline Number of channel per ASIC & 1 \\
\hline Polarity & negative \\
\hline Dynamic range in charge & $\mathrm{Q}=25 \mathrm{fC}-1.2 \mathrm{nC}$ \\
\hline Dynamic range in current peak & $\mathrm{Q}=1.25 \mu \mathrm{A}-60 \mathrm{~mA}$ \\
\hline Rise time of the current peak & $t_{r}=4 \mathrm{~ns}$ \\
\hline Fall time of the current peak & $t_{f}=36 \mathrm{~ns}$ \\
\hline Noise (RMS) & $<12 \mathrm{fC}$ \\
\hline Power & as low as possible \\
\hline Power supply & $1.6 \mathrm{~V}$ \\
\hline Output & word of 12 bits at $40 \mathrm{MHz}$ \\
\hline
\end{tabular}

The overall layout of the latest ASIC iteration, FATALIC5, is shown in Fig. 2. The first stage of FATALIC is a current conveyor which splits the input signal into three ranges with gain ratios of 1,8 and 64 . Each current conveyor output is followed by a shaper and a dedicated pipelined 12-bit Analogue-to-Digital Converter (ADC) operating at $40 \mathrm{MHz}$. As a result, the analogue signal from the physics events is digitised at the ADC output, which in case for FATALIC is integrated in the ASIC itself. Due to bandwidth limitations, only two gains are forwarded to the output. Auto-selection of the data to be transmitted is performed between the high and the low gains, while the medium gain data are always included. This auto-selection is performed digitally inside the chip based on the saturation of the most sensitive channel. Fig. 3 shows an example of an analogue signal at the FATALIC shaper output (using a digital scope) and a digitised event at the ADC output, both shown for a muon event.

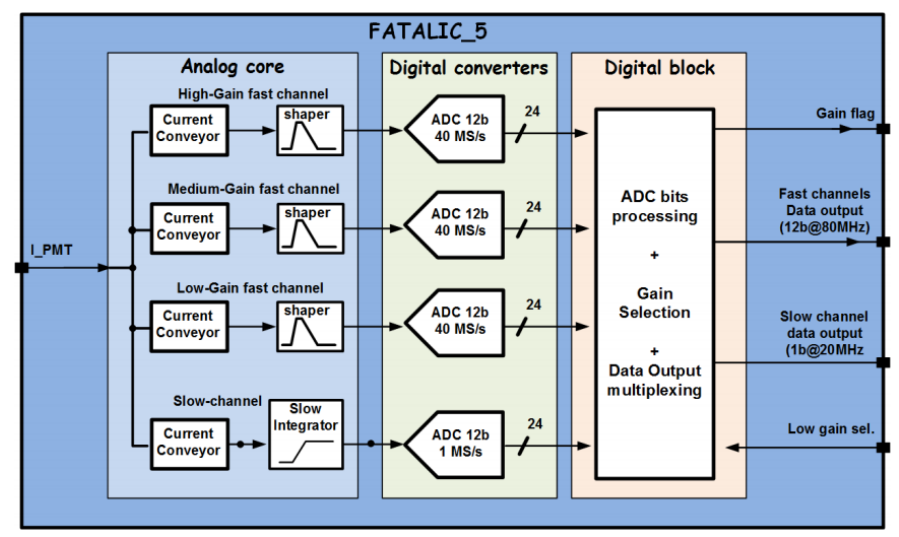

Fig. 2. Block diagram of the latest design of FATALIC ASIC.
The dynamic performance of the whole chain is measured in terms of resolution using the dedicated charge injection system designed for calibration. Fig. 4 shows the linearity results for the three gains of the previous FATALIC ASIC, FATALIC4b. It can be seen that the non-linearity is contained within $2 \%$ for the high and medium gains over their whole range. However, saturation is observed for the low gain starting at around 600 $\mathrm{pC}$.

This drawback has been addressed in FATALIC5. The improvement of the linearity, particularly for the low gain, is achieved at the input stage (the current conveyor) by reducing the impact of the high input currents (about $60 \mathrm{~mA}$ for a charge of $1.2 \mathrm{nC}$ ) on the Grid-Source voltage variations of the input transistor. Use of larger transistors allows these variations to be reduced by almost one order of magnitude. Fig. 5 gives the linearity results for the latest FATALIC5 design, obtained using simulation. The non-linearity is now better than $1 \%$ over the whole dynamic range for all gains.

Another improvement implemented in FATALIC5 is the addition of the slow channel (Fig. 2), necessary for the measurement of low current. This measurement is needed for the absolute detector calibration with radioactive caesium source, which produces a known but low signal. While the three previously described channels have integration times of $25 \mathrm{~ns}$ (the LHC clock period), the slow channel has a $100 \mu$ s time constant inside the integrator. For this channel, the digitisation is performed over $1.2 \mu \mathrm{s}$ by the ADC, and the integration time is $10 \mathrm{~ms}$. Larger integration times are accessible by digital means, when requested. The fast and slow channels are always DC coupled, and the current is split by allocating $20 \%$ to the fast channel, and $80 \%$ to the slow one. The optimisation of this ratio is obtained using simulation. The FATALIC5 design has been recently sent to foundry, and its first performance tests are planned for July 2017.

\section{Signal ReCONSTRUCTION}

\section{A. Optimal Filtering in TileCal}

Optimal Filtering (OF) is a method of reconstructing the amplitude of an analogue signal from its digital samples. It is currently used in TileCal as a main reconstruction method, and is also envisaged to be used in the future with one of the front-end readout options implemented. The method is welldocumented in the designated ATLAS note [5], and here we describe the main principle behind Optimal Filtering.

The digitised samples, such as those at the output of FATALIC, can be expressed as:

$$
S_{i}=p+A g\left(t_{i}+\tau\right)+n_{i}
$$

where $p$ is the signal pedestal, $A$ is the true amplitude, $g(t)$ is the normalized reference pulse shape (taken from the shaper circuit, which provides a stable and well-defined pulse shape), $\tau$ is the phase between the expected and measured pulse times, and $n_{i}$ is the noise term, which is typically modelled by a Gaussian distribution if only electronic noise is present. In order to make the phase $\tau$ an output parameter of the 


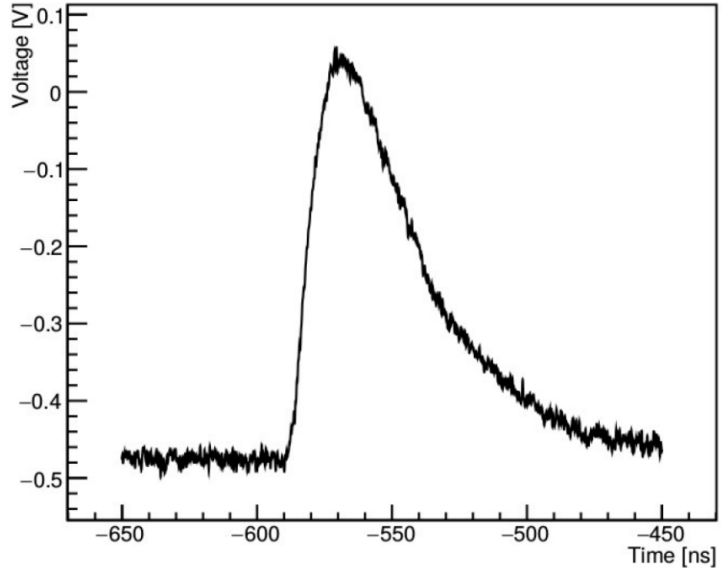

(a)

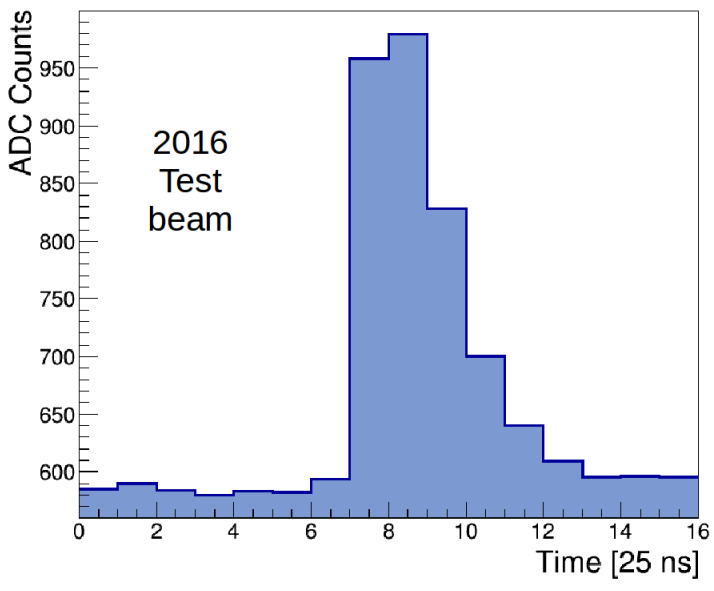

(b)

Fig. 3. (a) Analogue signal (shaper output) of a muon event. (b) Digital signal (ADC output) of a muon event through the whole chain.

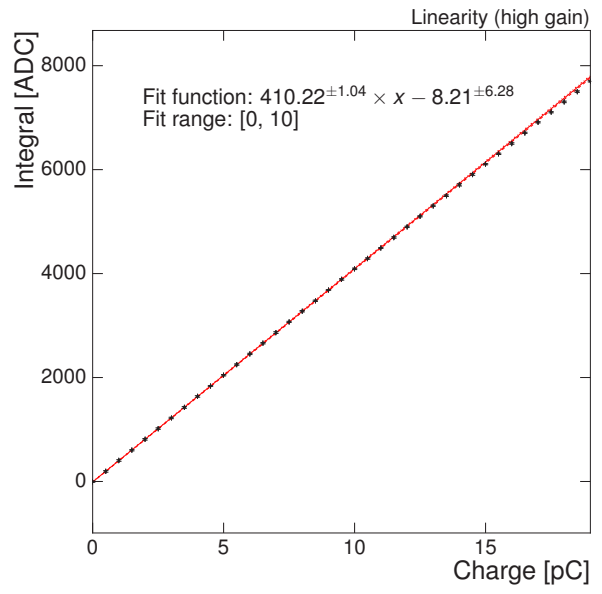

(a)

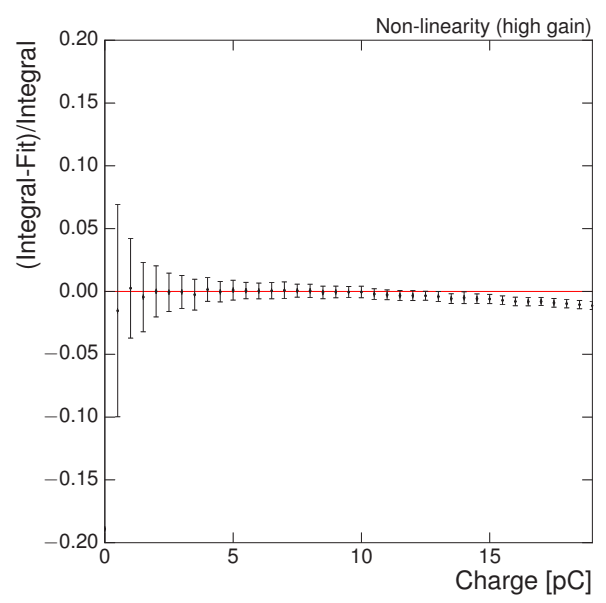

(d)

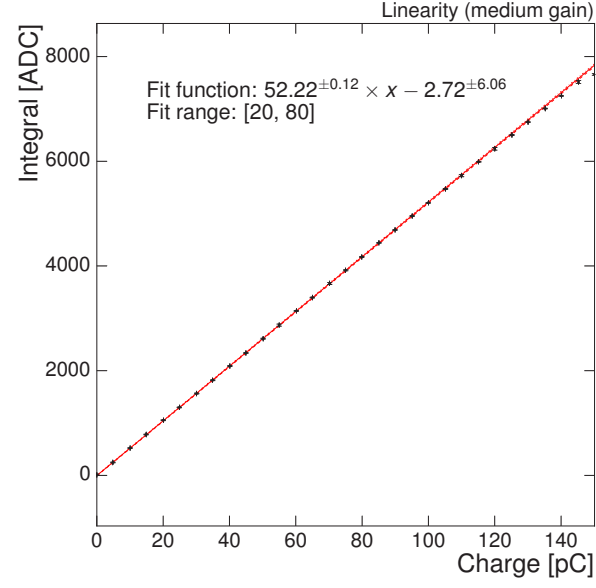

(b)

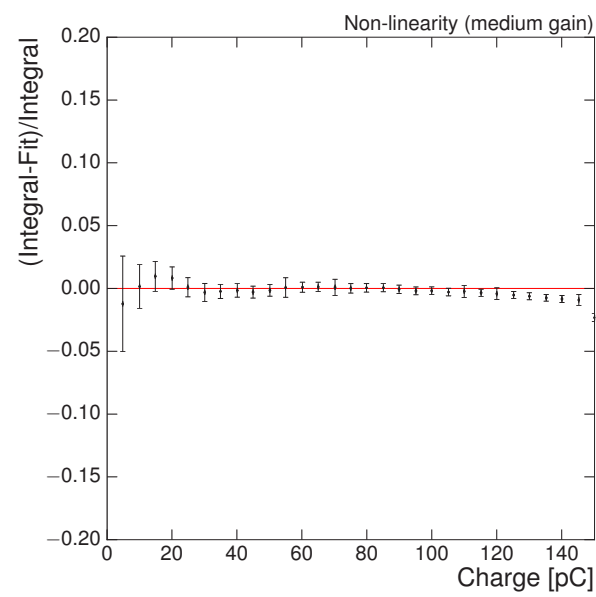

(e)

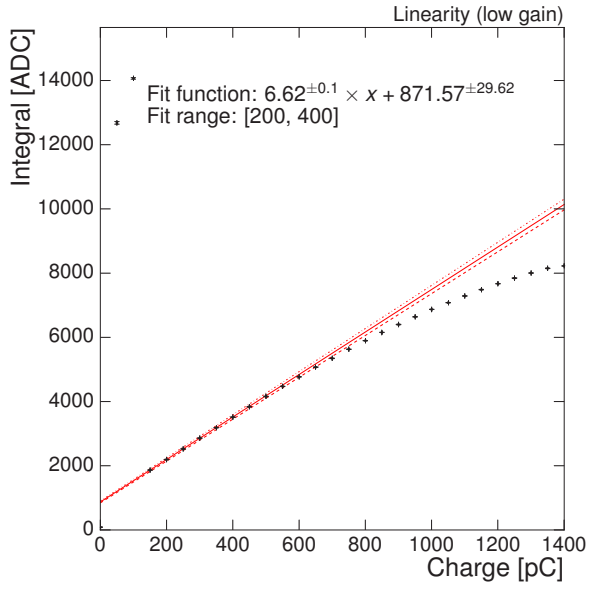

(c)

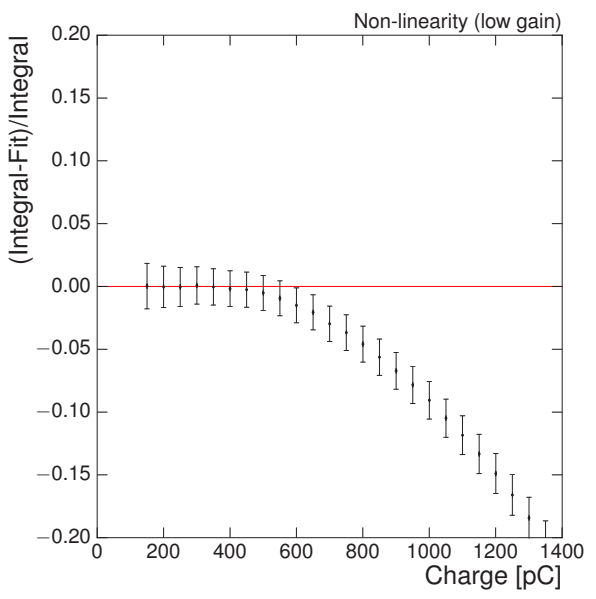

(f)

Fig. 4. Linearity and non-linearity of the previous design (FATALIC4b) for (a, d) high, (b, e) medium and (c, f) low gains. 


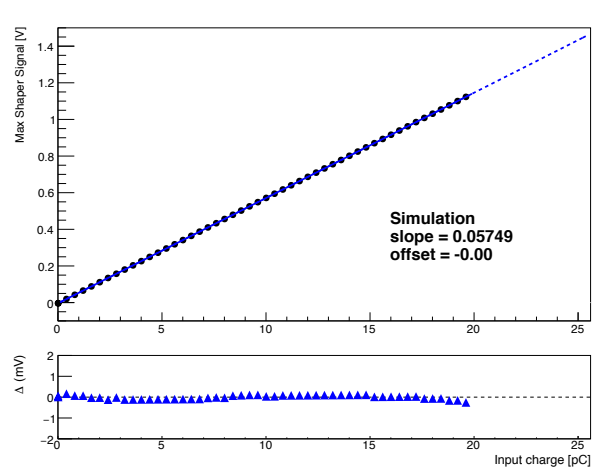

(a)

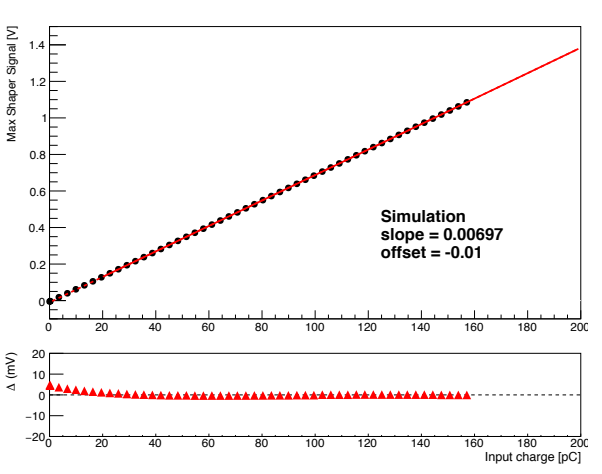

(b)

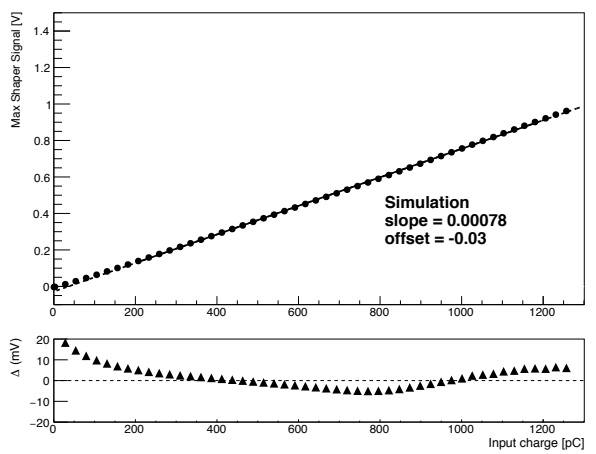

(c)

Fig. 5. Linearity of the latest design (FATALIC5) for (a) high, (b) medium and (c) low gains (simulation results). $\Delta$ (mV) in the bottom plots indicates the absolute difference between the shaper signal and the linear fit. $1 \mathrm{mV}$ corresponds to $0.1 \%$ relative difference with respect to the whole dynamic range.

algorithm, the dependence of $S_{i}$ on $\tau$ can be linearised via Taylor's expansion at first order:

$$
S_{i} \simeq p+A g\left(t_{i}\right)-A \tau g^{\prime}\left(t_{i}\right)+n_{i}=p+A g_{i}-A \tau g_{i}^{\prime}+n_{i}
$$

Three sets of free parameters $a_{i}, b_{i}$ and $c_{i}$ are defined, called OF weights, such as:

$$
A=\left\langle\sum_{i=1}^{N} a_{i} S_{i}\right\rangle=\sum_{i=1}^{N} a_{i}\left\langle S_{i}\right\rangle
$$

where $N$ is the number of samples.

The OF procedure aims at minimizing the variance of the linear combinations of these parameters subject to a set of constraints. These constraints lead to a set of linear equations [5], which prompt the derivation of the OF weights and hence the amplitude, pulse time and pedestal can be calculated.

\section{B. Optimal Filtering application to FATALIC}

1) Analytical pulse shape: In order to validate the principle of the Optimal Filtering reconstruction method, a study was performed with the reference pulse shape taken from the analytic function of the following form:

$$
V(t)=V_{\text {norm }} \times \frac{E r f\left(1-e^{-\frac{t-t_{\text {up }}}{\tau_{\text {up }}}}\right)+1}{2} \times e^{-\frac{t-t_{\text {down }}}{\tau_{\text {down }}}}+V_{0}
$$

where $V_{\text {norm }}$ is the normalisation parameter, $\operatorname{Erf}$ is the Gaussian error function [6], $t_{\text {up }}$ and $\tau_{\text {up }}$ are the pulse rise time parameters, $t_{\text {down }}$ and $\tau_{\text {down }}$ are the pulse fall time parameters, and $V_{0}$ is the pedestal. This function approximates the pulse shape measured with scope (Fig. 3, a), and is represented in Fig. 6 where it is compared with the scope output. The shape is clearly asymmetrical, making it different from the pulses of other front-end options (e.g. 3in1 [7]).

2) Construction of pulses: Before the OF method is tested in real conditions, it is important to validate its performance using known sampled pulses. In this study, to produce a sampled digitised pulse, the input reference pulse shape (Fig. 6) is normalised and sampled every $25 \mathrm{~ns}$ which is the LHC clock period. Then the normalised pulse is multiplied by the energy
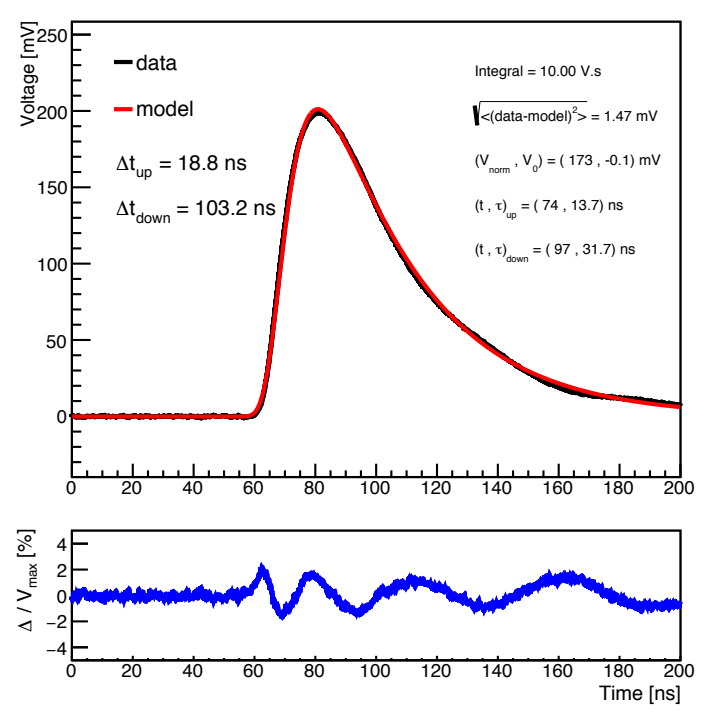

Fig. 6. Analytical pulse shape ("model") compared with the scope output ("data").

factor and added with a fixed typical pedestal value. The energy factor is obtained from a known conversion rate of the input charge to the energy deposited in a TileCal cell, which is taken from a uniform distribution. The noise factor is then added for each sample independently, uncorrelated between samples in the first approximation. The noise factors for each sample are considered as independent Gaussian distributions, RMS of which are later on referred to as noise levels.

As shown above, FATALIC layout comprises three different gains covering three dynamic ranges. While the medium gain is always present, high or low gain is selected depending on the saturation of either gain. For the purpose of validating the OF method with the analytical pulse shape whilst also simulating the gain switch behaviour, an effective digitised pulse is constructed by using the medium and alternative gain based on the following criteria: 
- if both high and medium gains are saturated $\left(s_{\mathrm{HG}}>4095\right.$ ADC, $s_{\mathrm{MG}}>4095$ ADC):

$$
-s_{\text {eff }}=64 \times s_{\mathrm{LG}} \mathrm{ADC}
$$

- if only high gain is saturated, and medium gain is not $\left(s_{\mathrm{HG}}>4095 \mathrm{ADC}, s_{\mathrm{MG}}<4095 \mathrm{ADC}\right)$ :

$$
-s_{\text {eff }}=8 \times s_{\mathrm{MG}} \mathrm{ADC}
$$

- if neither high nor medium gain are saturated $\left(s_{\mathrm{HG}}<\right.$ 4095 ADC, $s_{\mathrm{MG}}<4095$ ADC):

$$
-s_{\text {eff }}=s_{\mathrm{HG}} \mathrm{ADC}
$$

Here $s_{\mathrm{HG}}, s_{\mathrm{MG}}, s_{\mathrm{LG}}$ correspond to the signal output yields of the high, medium and low gains, and $s_{\text {eff }}$ is the resulting signal of an effective digitised pulse encoded in 18 bits. Fig. 7 shows the digitised pulses for the medium gain, alternative gain, and the effective 18-bit digitised pulse.

3) Performance results: The main performance criteria presented here is the pulse energy resolution, defined as follows:

$$
\frac{\Delta E}{E}=\frac{E_{\mathrm{OF}}-E_{\text {true }}}{E_{\text {true }}},
$$

where $E_{\text {true }}$ is the true energy, and $E_{\mathrm{OF}}$ is the energy reconstructed by the Optimal Filtering method.

The energy resolution plots for a typical LHC energy range from $100 \mathrm{MeV}$ to $1.5 \mathrm{TeV}$ are shown in Fig. 8 for two different scenarios: with and without the electronic noise. For the latter case, the typical noise levels are considered: 3.5 ADC $(\sim 8.4$ fC) for the high gain, 1.5 ADC ( $\sim 29 \mathrm{fC})$ for the medium gain, and 1.5 ADC ( $\sim 230 \mathrm{fC})$ for the low gain. It can be seen that the energy resolution is kept within percentage level across four orders of magnitude of the signal energy.

\section{RESULTS WITH REAL DATA}

For the final validation of the Optimal Filtering method and the whole chain of the FATALIC readout system, the signal reconstruction performance was tested with real data collected during a preliminary beam test at CERN [8]. In Fig. 9 (a) the OF method is compared with the flat estimation method, which is the basic energy reconstruction corresponding to the simple summation of digitised samples. Clearly, the Optimal Filtering reconstruction provides a significant improvement of energy resolution compared to the simple summation approach. Fig. 9 (b) shows the two-dimensional plot of the energy reconstructed using the OF method for muon events recorded by two different channels of a single cell, and the expected correlation is observed accordingly.

\section{CONCLUSION}

We have presented an overview and performance of the FATALIC front-end readout option of the TileCal HighLuminosity LHC upgrade foreseen in 2024. The characterisation of the full chain was shown, as well as the signal reconstruction was validated. The Optimal Filtering signal reconstruction method was introduced, its adaptation to fully exploit the FATALIC three-range layout was presented and its performance results were given by using both analytical pulse shapes and the real data from the beam test at CERN.

\section{REFERENCES}

[1] ATLAS Collaboration, "The ATLAS Experiment at the CERN Large Hadron Collider," JINST, vol. 3, p. S08003, 2008.

[2] ATLAS tile calorimeter: Technical Design Report, 1996, no. CERNLHCC-96-042. [Online]. Available: https://cds.cern.ch/record/331062

[3] L. Rossi and O. Brüning, "High Luminosity Large Hadron Collider A description for the European Strategy Preparatory Group," Tech. Rep. CERN-ATS-2012-236, 2012. [Online]. Available: https://cds.cern.ch/record/1471000

[4] L. Royer, "FATALIC: A Dedicated Front-End ASIC for the ATLAS TileCal Upgrade," Oct 2015. [Online]. Available: https://cds.cern.ch/record/2057101

[5] E. Fullana, J. Castelo, V. Castillo, C. Cuenca, A. Ferrer, E. Higón, C. Iglesias, A. Munar, J. Poveda, A. Ruiz-Martinez, B. Salvachúa, C. Solans, R. Teuscher, and J. Valls, "Optimal Filtering in the ATLAS Hadronic Tile Calorimeter," Tech. Rep. CERN-ATL-TILECAL-2005-001, 2005. [Online]. Available: https://cds.cern.ch/record/816152

[6] E. W. Weisstein, "Erf. From MathWorld-A Wolfram Web Resource," last visited on 24/5/2017. [Online]. Available: http://mathworld.wolfram.com/Erf.html

[7] K. Anderson et al., "Design of the front-end analog electronics for the ATLAS tile calorimeter," Nucl. Instrum. Meth., vol. A551, pp. 469-476, 2005.

[8] M. Volpi, L. Fiorini, and I. Korolkov, "Time inter-calibration of hadronic calorimeter of ATLAS with first beam data." Tech. Rep. ATL-TILECAL-INT-2009-003, Nov 2009. [Online]. Available: https://cds.cern.ch/record/1223049 


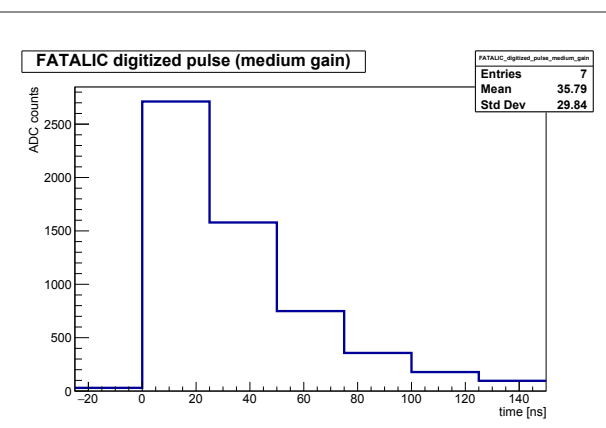

(a)

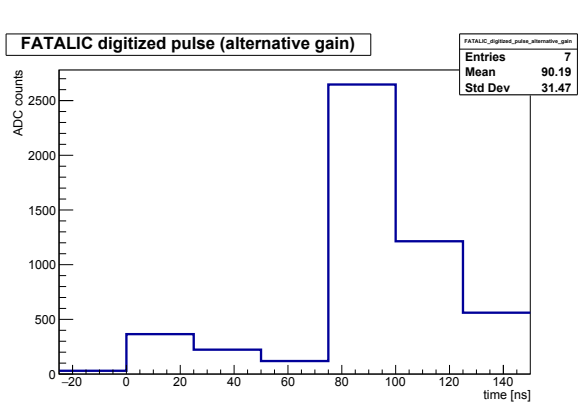

(b)

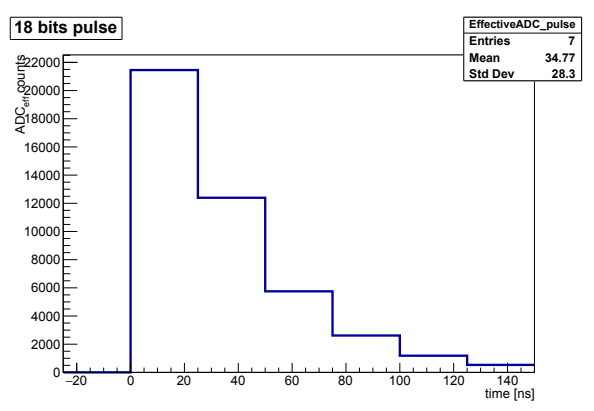

(c)

Fig. 7. FATALIC digitised pulse for (a) medium gain, (b) alternative gain, and (c) effective 18-bit digitised pulse.

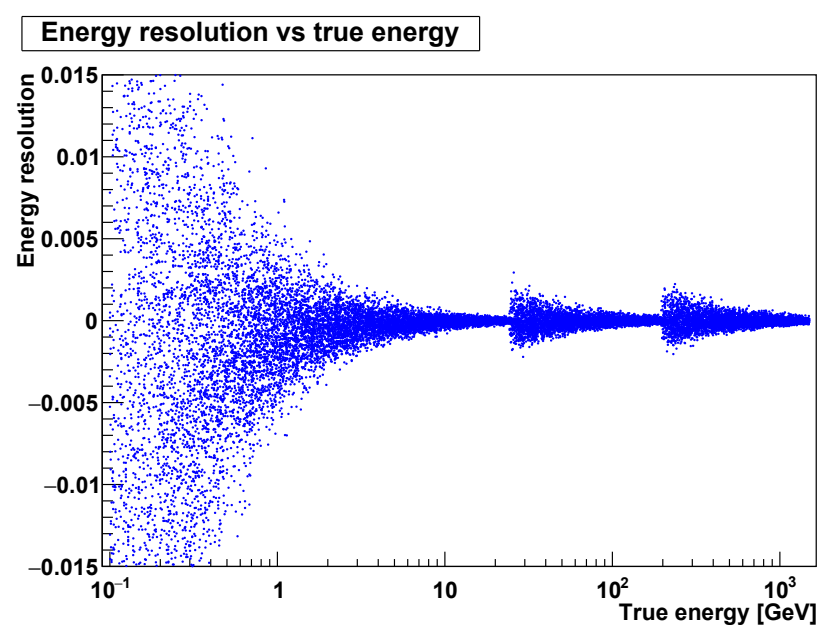

(a)

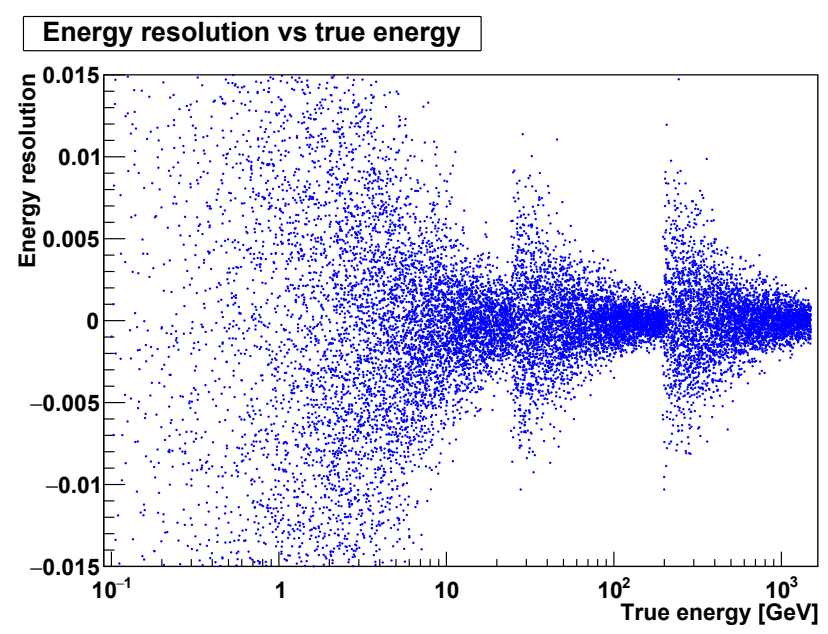

(b)

Fig. 8. FATALIC energy resolution with respect to the true pulse energy using analytical pulse shape: (a) without electronic noise, (b) with typical noise.

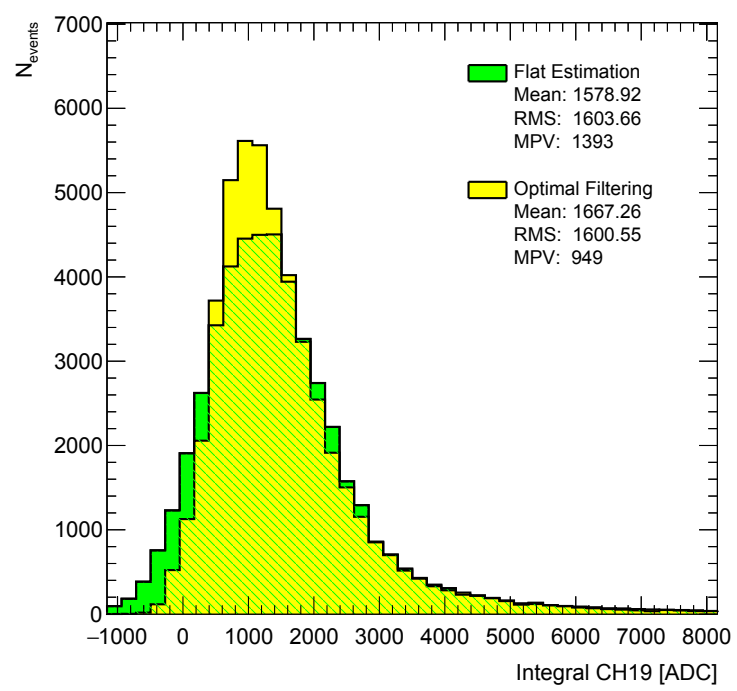

(a)

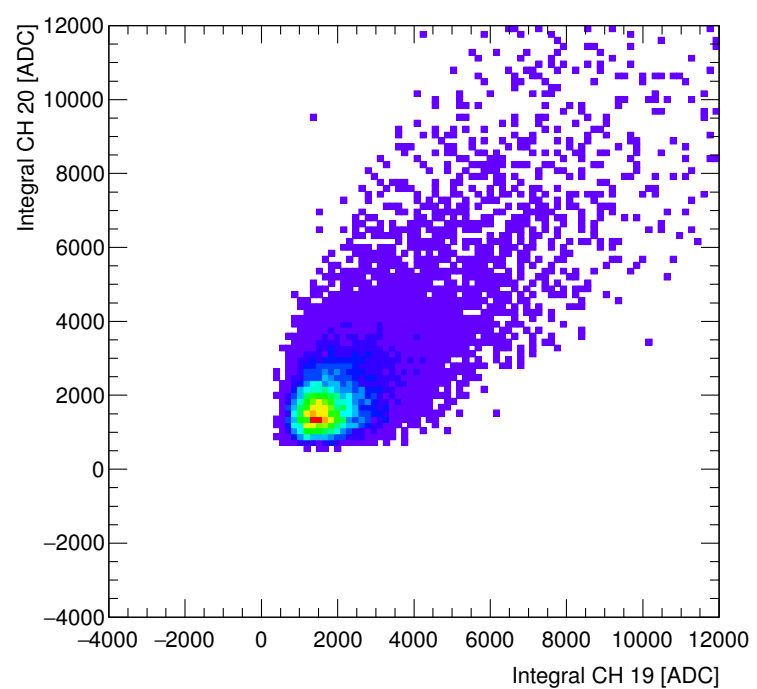

(b)

Fig. 9. Energy reconstructed using the real test beam data: (a) comparison of the OF method with the simple flat estimation method, (b) energy reconstructed using the OF method for muon events recorded by two different channels of a single cell. 